ARTICLE

DOI: $10.1038 / s 41467-017-01404-8$

OPEN

\title{
Ultra-antireflective synthetic brochosomes
}

\author{
Shikuan Yang ${ }^{1,2,3}$, Nan Sun ${ }^{1,2}$, Birgitt Boschitsch Stogin ${ }^{1,2}$, Jing Wang ${ }^{1,2}$, Yu Huang ${ }^{1,2}$ \& Tak-Sing Wong ${ }^{1,2,4}$
}

Since the early discovery of the antireflection properties of insect compound eyes, new examples of natural antireflective coatings have been rare. Here, we report the fabrication and optical characterization of a biologically inspired antireflective surface that emulates the intricate surface architectures of leafhopper-produced brochosomes-soccer ball-like microscale granules with nanoscale indentations. Our method utilizes double-layer colloidal crystal templates in conjunction with site-specific electrochemical growth to create these structures, and is compatible with various materials including metals, metal oxides, and conductive polymers. These brochosome coatings (BCs) can be designed to exhibit strong omnidirectional antireflective performance of wavelengths from 250 to $2000 \mathrm{~nm}$, comparable to the state-of-the-art antireflective coatings. Our results provide evidence for the use of brochosomes as a camouflage coating against predators of leafhoppers or their eggs. The discovery of the antireflective function of BCs may find applications in solar energy harvesting, imaging, and sensing devices.

\footnotetext{
${ }^{1}$ Department of Mechanical and Nuclear Engineering, The Pennsylvania State University, University Park, PA 16802, USA. ${ }^{2}$ Materials Research Institute, The Pennsylvania State University, University Park, PA 16802, USA. ${ }^{3}$ Institute for Composites Science Innovation, School of Materials Science and Engineering, Zhejiang University, Hangzhou 310027, China. ${ }^{4}$ Department of Biomedical Engineering, The Pennsylvania State University, University Park, PA 16802, USA. Correspondence and requests for materials should be addressed to S.Y. (email: shkyang@zju.edu.cn) or to T.-S.W. (email: tswong@psu.edu)
} 
$\mathrm{N}$ atural surfaces have demonstrated how different micro/ nanoscale surface architectures can yield an array of distinct interfacial functions ${ }^{1-9}$. While many of these surface structures can now be manufactured using advanced manufacturing techniques ${ }^{10-13}$, scalable fabrication methods capable of producing a number of these natural structures have remained elusive. Among these natural structures are leafhopperproduced brochosomes (Fig. 1) ${ }^{14-16}$. Naturally occurring integumental brochosomes are microscale granules with nanoscale surface indentations arranged in a honeycomb pattern, making the geometry of a brochosome particle similar to those of a soccer ball $^{17}$. Leafhoppers living in different regions create brochosomes with significantly varied structural geometries, with distinct diameters and numbers of pits ${ }^{18}$. In addition to their use as nonsticking coatings ${ }^{19,20}$, the intricate nanoscale architecture and three-dimensional periodicity of these BCs suggest they may have complex optical properties. Interestingly, Swain proposed in 1936 that these brochosomes might serve as a camouflage coating to hide the eggs of leafhoppers from their predators or parasites ${ }^{21}$, but no experimental evidence has been shown thus far. This is due to the fact that the optical functions of the BCs remained minimally understood as large quantities of brochosomes for systematic study are not readily producible. Until now, micro/ nanomanufacturing techniques to create brochosomes of various geometries and material compositions have not been available.

Here, we develop a fabrication concept for preparing BCs composed of closely packed artificial brochosomes, whose shape and geometry closely mimic the natural ones created by leafhoppers. We utilize double-layer colloidal crystal (DCC) templates combined with site-specific electrochemical growth to prepare artificial BCs comprising metals, metal oxides, polymers, or their hybrids on any conductive substrates. The structure of the synthetic BCs, defined by the diameter of the brochosomes, the inter-brochosome distance, as well as the size and depth of the pits within the brochosomes, can be precisely engineered, allowing us to systematically explore their structure-property relationships. Using silver (Ag) as a model material, we have shown that $2-\mu \mathrm{m}$ thick Ag BCs (i.e., BCs comprise $2 \mu \mathrm{m}$ diameter brochosomes) are capable of reflecting $<\sim 1 \%$ on average of any wavelength in the $250-2000 \mathrm{~nm}$ optical window. This reflectance is comparable to those of the state-of-the-art synthetic antireflective materials ${ }^{22}$. The superior antireflection is attributed to the unique structural geometries of the brochosomes, as demonstrated experimentally and numerically. Additionally, our experimental results suggest a possible use of BCs as a camouflage and protective layer for leafhoppers or their eggs against potential predators in their natural habitats ${ }^{18,23,24}$.

\section{Results}

Fabrication of synthetic brochosomal coatings. Our synthetic BCs were created by first preparing a highly ordered DCC template, followed by site-specific electrodeposition on the template (Fig. 2a-d). The DCC template was prepared through a layer-bylayer stacking process (Fig. 2e). First, a monolayer colloidal crystal (MCC) template $\left(>2 \mathrm{~cm}^{2}\right)$ composed of polystyrene (PS) spheres was prepared by spin-coating, and was then transferred onto the water/air interface (Process I in Fig. 2e) ) $^{25-27}$. An arbitrary substrate was used to pick up the free-standing MCC template from underneath (Process II in Fig. 2e, b). The substrate could be in the form of a smooth, flexible, curved, or roughened surface. A thin layer of $\mathrm{Au}$ film $(\sim 100 \mathrm{~nm})$ was then deposited onto the MCC template (Process I in Fig. 2a). Next, another layer of MCC template comprising smaller PS spheres was transferred onto the Au-coated MCC template (Process III in Fig. 2e), yielding the DCC template (Process II in Fig. 2a, c), which was
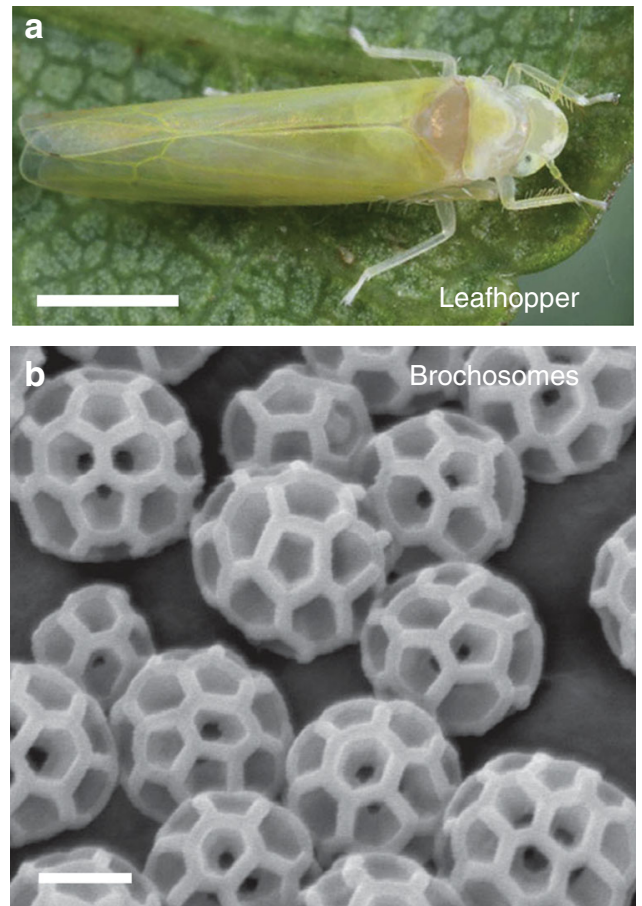

Fig. 1 Leafhopper and its brochosomes. a Optical image of a leafhopper, Alnetoidia alneti (Dahlbom), and $\mathbf{b}$ an electron micrograph showing its brochosomes. Both images are reprinted from ref. ${ }^{19}$, by permission of the Royal Society. Scale bars, $1 \mathrm{~mm}$ (leafhopper) and $200 \mathrm{~nm}$ (brochosomes)

then used as the working electrode to perform electrochemical deposition of targeting materials. The electrodeposited material was constrained to grow conformally on the large PS spheres in the bottom layer template and continue to grow from the Au seed layer up and around the small PS spheres in the top layer template. After removing the PS spheres, BCs of the targeting materials can be obtained (Process III in Fig. 2a, d). The final structures closely mimic those of the natural brochosomes.

We used Ag as a model structural material to investigate the fabrication parameters of the synthetic BCs (Fig. 3). Adopting the DCC templating method, Ag BCs with a uniform structure can be prepared over a large area $\left(>5 \mathrm{~cm}^{2}\right)$. For example, synthetic Ag brochosomes with a diameter of $\sim 2 \mu \mathrm{m}$ were arranged in a hexagonally close-packed configuration (Fig. 3a). On this particular surface, there are $\sim 19$ pits hexagonally close-packed over the curved spherical surface (Fig. 3b), with the opening size and pit depth of 470 and $225 \mathrm{~nm}$, respectively (Fig. 3c). This configuration is distinctively different from the conventional binary colloidal crystal templates ${ }^{28}$, which will typically result in $\sim 12$ pits located at the equators of the large spheres (Supplementary Fig. 1).

The availability of PS spheres of various sizes (from $<100 \mathrm{~nm}$ to $>10 \mu \mathrm{m}$ ) allows us to design synthetic BCs with a broad range of structural geometries (Supplementary Note 1). Using our technique, the pit number and size can be readily tailored by using PS spheres with different sizes to form the top layer MCC template (Supplementary Figs. 2, 3). The depth of the pits can be controlled by the electrodeposition time (Fig. 3d). Specifically, we found that the low growth rate at a low deposition voltage (e.g., $1.5 \mathrm{~V}$ ) is preferred in order to accurately control the pit depth (Supplementary Figs. 4-7). In addition, we have demonstrated the formation of BCs using PS beads with diameters ranging from 1 to $4.5 \mu \mathrm{m}$ with various pit sizes (Supplementary Figs. 8-10). Given the sizes of the PS spheres at the top and bottom layers of the DCC template, the geometrical parameters of the synthetic 

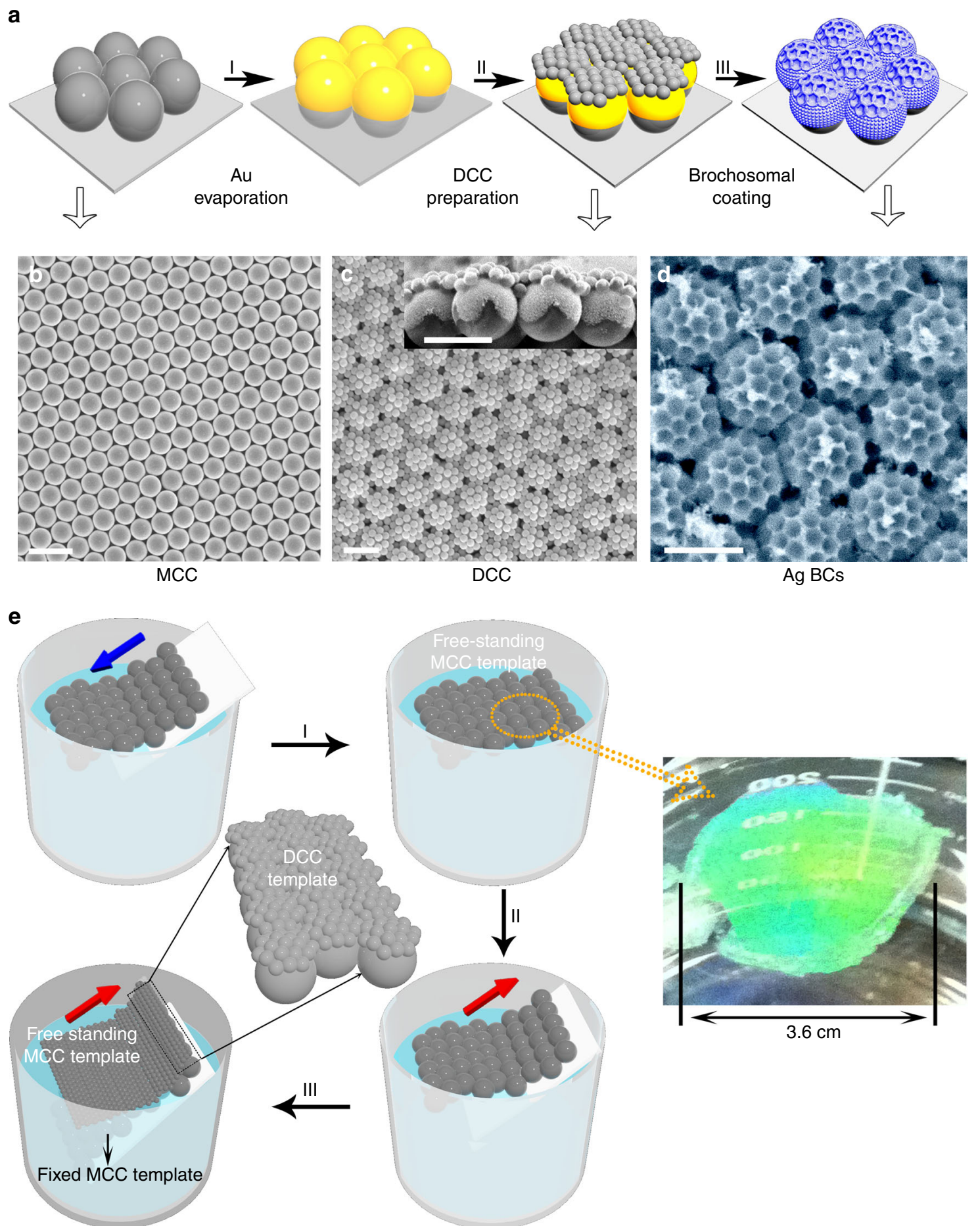

Fig. 2 Fabrication of synthetic brochosomal coating. a Fabrication process of the synthetic BCs. I. Gold evaporation. II. Preparation of DCC template. III. DCC template-based site-specific electrochemical deposition which creates BCs after PS spheres removal. b-d SEM images of MCC template (scale bar, 2 $\mu \mathrm{m}$ ), DCC template (scale bar, $2 \mu \mathrm{m}$, and inset, $2 \mu \mathrm{m}$ ), and Ag BCs (scale bar, $2 \mu \mathrm{m}$ ), respectively. e Fabrication process of DCC template. I. MCC template transferred onto the air/water interface. II. MCC template transferred onto another arbitrary substrate. III. DCC template created by transferring another layer of MCC template onto a pre-fabricated MCC template that was thermally anchored on a substrate. Note that the thermal treatment of the MCC template around the glass transition temperature increases the interfacial area, thereby enhancing the binding between the PS spheres and the silicon substrate

BCs can be precisely controlled. These geometrical parameters include the opening size $(2 r)$ and depth $(h)$ of the pits, as well as the number of pits $(n)$. We can predict $h$ using the following equation (equation describing the height of a spherical cap): $h=R_{\mathrm{t}}-\left(\sqrt{R_{t}^{2}-r^{2}}\right)$ if $h<R_{\mathrm{t}}$, where $R_{\mathrm{t}}$ is the radius of the top layer small PS spheres (Supplementary Fig. 11). In addition, the pit size $\left(2 R_{\mathrm{t}}\right)$, pit number $(n)$, and the diameter $\left(2 R_{\mathrm{b}}\right)$ of the brochosomes can be related by $n \cong \frac{\pi}{2 \sqrt{3}}\left(\frac{R_{\mathrm{b}}}{R_{\mathrm{t}}}\right)^{2}$, which agrees well with the experimental results (Fig. 3e). 

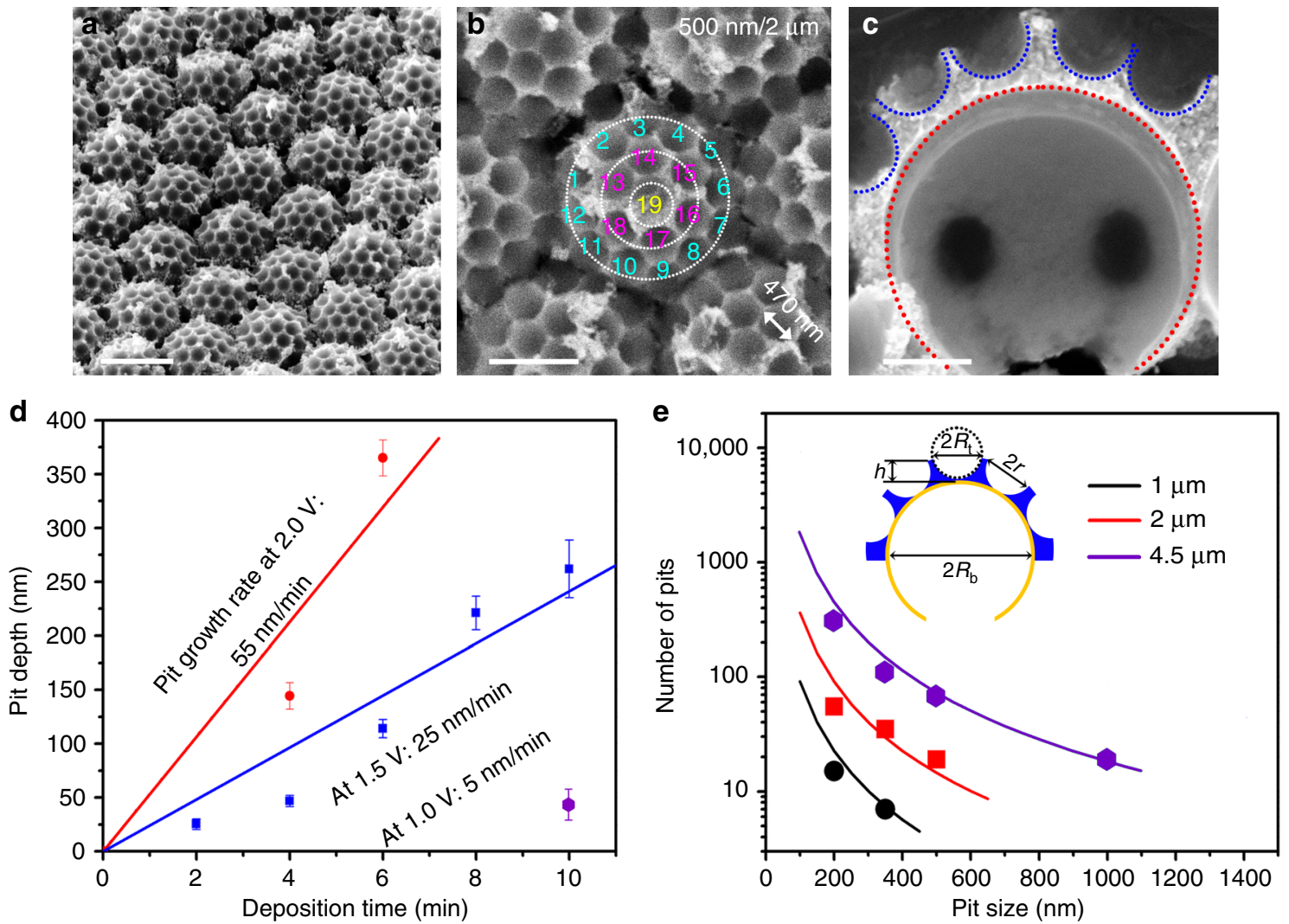

Fig. 3 Structural control of BCs. a, b SEM images of Ag BCs at different magnifications. Scale bars: (a) $2 \mu \mathrm{m}$, and (b) $1 \mu \mathrm{m}$. c Cross-sectional image of a silver brochosome particle. Scale bar: $500 \mathrm{~nm}$. d A plot showing the growth rate of pit depth at different electrodeposition voltages. Error bars represent standard deviations from 100 independent measurements of the pits. e A plot showing the relationship between the number of pits and the pit size on the brochosome particles for a given size of bottom layer spheres (labeled in inset). Solid lines indicate theoretical predictions. Inset showing the schematic of the relevant geometrical parameters of a brochosome-like particle

Optical characterizations. Depending on the size of PS spheres in the top layer template, the as-deposited Ag BCs (with PS spheres) display different colors (Fig. 4a). The Ag BCs become dark in color after dissolving the PS spheres. The reflection spectra revealed that the reflection is continuously reduced as the pit depth is increased until $h=0.55 R_{\mathrm{t}}$ (Supplementary Fig. 12). Unlike planar metals which behave as excellent mirrors, our BCs consist of metal nanoparticle assemblies in the size range of $50-100 \mathrm{~nm}$, which can further suppress reflection due to absorption by plasmon excitations ${ }^{29,30}$. The specular reflection on our BCs at an incident angle of $45^{\circ}$ in the ultraviolet (UV) $(250-380 \mathrm{~nm})$, visible (Vis) $(380-780 \mathrm{~nm})$, and near infrared (NIR) $(780-2000 \mathrm{~nm})$ region is below $0.4 \%, 0.2 \%$, and $1.4 \%$, respectively, when the pit opening size is $\sim 470 \mathrm{~nm}$ with a depth of $\sim 260 \mathrm{~nm}$, respectively (Fig. 4b). These values are substantially lower than those of the control samples, including a thermally evaporated planar Ag membrane, an Ag nanoporous film (Supplementary Fig. 13) prepared using the same electrodeposition method, and a planar Ag mesoporous film embedded with honeycomb arranged pits (Supplementary Fig. 14).

The antireflection of the $2 \mu \mathrm{m}$ brochosome arrays can be further improved by modifying the pit size (Fig. $4 \mathrm{c}$ and Supplementary Note 2). In particular, optimized antireflection properties in the $250-2000 \mathrm{~nm}$ wavelength range can be achieved when the ratio of the pit depth and the diameter of the pitcreating PS spheres $h / R_{\mathrm{t}}$ is 0.6 according to FDTD simulation results $^{31}$ (Supplementary Figs. 15, 16), which agrees well with the experimental results (i.e., $h / R_{\mathrm{t}} \sim 0.55$ ) (Supplementary Fig. 12). The optimal pit depth to size ratio ensures efficient trapping of light by multiple internal reflections within the pit to suppress overall reflection of the coating ${ }^{32,33}$. In addition, our FDTD simulation results show that having a large number of pits (i.e., $n$ $>7$ ) on individual brochosomes is essential to further enhance the antireflection performance of the BCs (Supplementary Fig. 17), consistent with our experimental observations (i.e., $n>10$ ), suggesting that more pits per unit projected surface area are desired to suppress reflection.

The antireflection property of the $\mathrm{BCs}$ is insensitive to the angle of the incident light (Fig. 4d and Supplementary Fig. 18). Specifically, as the incident angle varies from $8^{\circ}$ to $45^{\circ}$, the reflectance from the BCs $\left(R_{\mathrm{b}}=1 \mu \mathrm{m}, R_{\mathrm{t}}=250 \mathrm{~nm}, h=260 \mathrm{~nm}\right)$ is $<0.25 \%$ in the visible region and $<1.5 \%$ in the $700-2000 \mathrm{~nm}$ region. For an incident angle as large as $65^{\circ}$, the BCs still maintain low reflectance of $<0.7 \%$ in the visible region, and $<8 \%$ in the wavelength region of $700-2000 \mathrm{~nm}-\mathrm{a}$ performance that is comparable to the state-of-the-art synthetic antireflective coatings $^{22}$ (Supplementary Fig. 19). In comparison, the reflection spectrum of the planar mesoporous Ag film composed of hexagonally arranged nanovoids (equivalent to $R_{\mathrm{b}} \rightarrow \infty, R_{\mathrm{t}}=$ $250 \mathrm{~nm}, h=\sim 250 \mathrm{~nm}$, see Supplementary Fig. 14) demonstrated angle dependence (Supplementary Fig. 20). This further shows that the radius of curvature (i.e., $R_{\mathrm{b}}$ ) of the individual synthetic brochosomes where the distributed pits are located is important for enhanced omnidirectional antireflection. Overall, our experimental measurements showed that strong omnidirectional antireflection (i.e., reflectance $<10 \%$ ) of BCs can be attained when $\sim 9 \lambda>R_{\mathrm{b}}>\sim 1.4 \lambda$ ( $\lambda$ is wavelength of the electromagnetic waves), $\sim 2 \lambda>R_{\mathrm{t}}>\sim 0.5 \lambda$, and $R_{\mathrm{t}} / R_{\mathrm{b}}<0.35$ (or $n>\sim 7$ ) (Supplementary Figs. 21, 22). Therefore, the pit geometry $\left(R_{\mathrm{t}}, h\right)$ and number $(n)$, as well as the brochosome size $\left(R_{\mathrm{b}}\right)$ are important 
a

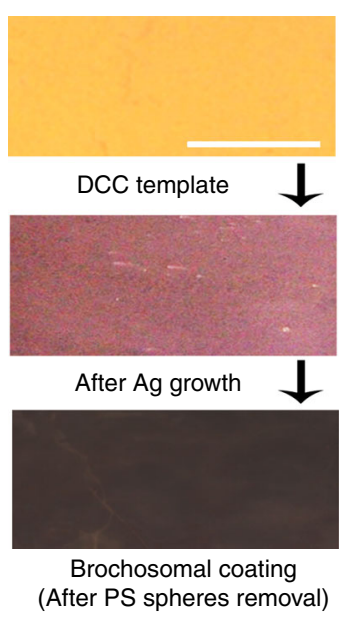

C

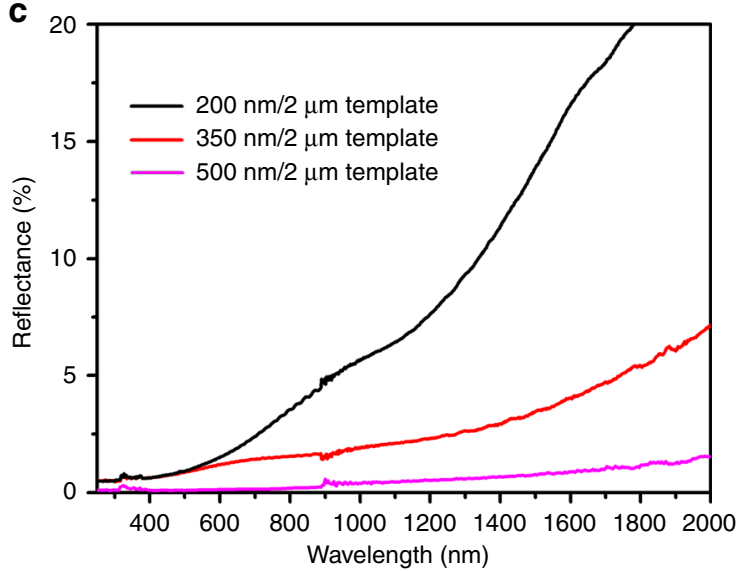

b
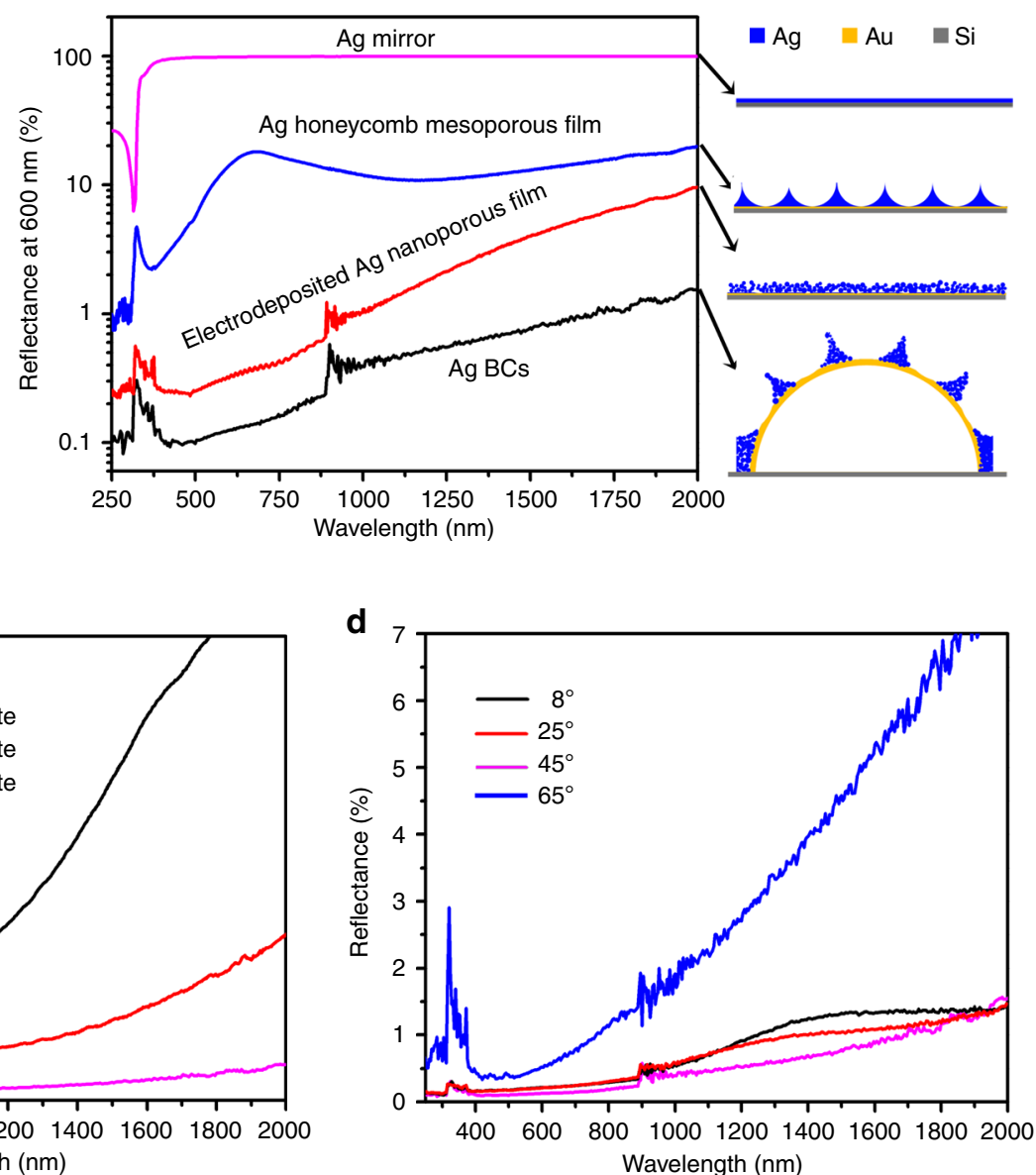

Fig. 4 Antireflection performance of the synthetic BCs. a Optical images of DCC template before and after Ag electrochemical growth, and after the removal of PS beads. Scale bar: $5 \mathrm{~mm}$. b A plot showing the reflection spectra of $\mathrm{Ag}$ mirror (in magenta), $\mathrm{Ag}$ honeycomb mesoporous film (in blue), $\mathrm{Ag}$ nanoporous film (in red) and $\mathrm{Ag} \mathrm{BCs}$ (in black). Schematic drawings of the corresponding structures are shown on the right. The Ag BCs were prepared by using the DCC template composed of $2 \mu \mathrm{m}$ large PS spheres with the top surface covered by $500 \mathrm{~nm}$ PS spheres. After electrodeposition, the pits of these $B C s$ were $470 \mathrm{~nm}$ in opening size and $260 \mathrm{~nm}$ in depth. The Ag honeycomb mesoporous film was prepared by electrodeposition using $500 \mathrm{~nm}$ PS spheres on an Au-covered silicon wafer. The depth of the pits was $250 \mathrm{~nm}$ (Supplementary Fig. 14). c A plot showing the reflection spectra of BCs with different pit sizes. d A plot showing the angle dependence of the reflection spectra of the Ag BCs composed of $2 \mu \mathrm{m}$ particles with pits $470 \mathrm{~nm}$ in opening size and $260 \mathrm{~nm}$ in depth

design parameters to achieve best omnidirectional antireflection of the BCs.

\section{Discussion}

The ultra-antireflective property of the synthetic BCs at the UV and visible light range may suggest that their natural counterparts could have been optimized for antireflective and camouflage functions against leafhoppers' predators (e.g., birds or insects), whose active vision spectra are also in the UV and visible light range $^{34}$. To demonstrate the possible camouflage function of the $B C s$, we placed the synthetic BCs next to various leaf species (i.e., Chrysanthemum, Lantana, Callicarpa, and Fushia), and compared their colors through the simulated visions of a ladybird beetle (a predator of leafhopper) ${ }^{35}$. Note that the active vision spectrum of a ladybird beetle is from 311 to $605 \mathrm{~nm}^{36}$. Based on the images generated by the simulated vision of a ladybird, the appearance of the BCs and the green leaves have very high level of similarity both qualitatively (Fig. 5a) and quantitatively (Supplementary Fig. 23). To further support our hypothesis, we gathered the geometrical parameters of a number of representative natural integumental BCs, and found that their geometries (i.e., $R_{\mathrm{b}}$ and $R_{\mathrm{t}}$ ) are within the design parameters for strong omnidirectional antireflectance $(<10 \%)$ of the synthetic BCs (Fig. 5b and Supplementary Fig. 24). This further suggests that the natural and synthetic BCs may possess similar antireflective properties, which could be important survival functions that protect leafhoppers or their eggs from being detected by their predators in their natural environments ${ }^{18,23,24}$.

Finally, the fabrication concept of synthetic BCs is very general and can be extended to other material systems compatible with electrochemical deposition technique (Fig. 5c). For example, BCs of nickel $(\mathrm{Ni})$, gold $(\mathrm{Au})$, manganese oxide $\left(\mathrm{MnO}_{2}\right)$, and polypyrrole (PPy) can be prepared, where these materials have broad applications in energy and sensing applications ${ }^{37,38}$ (Supplementary Figs. 25-29). In addition, BCs of hybrid materials, for instance, Ag/ $\mathrm{MnO}_{2}$ can be prepared by a step-by-step electrodeposition method (Supplementary Fig. 29). Translating the highly complex geometries of natural brochosomes into materials architectures of various compositions may lead to advanced applications in solar energy harvesting, imaging, and sensing devices ${ }^{39-41}$.

\section{Methods}

Preparation of DCC template. Glass slides $(2.5 \times 7.5 \mathrm{~cm})$ were treated in a plasma cleaner (Harrick Plasma, PDC-32G) for 10 min to obtain a highly hydrophilic 

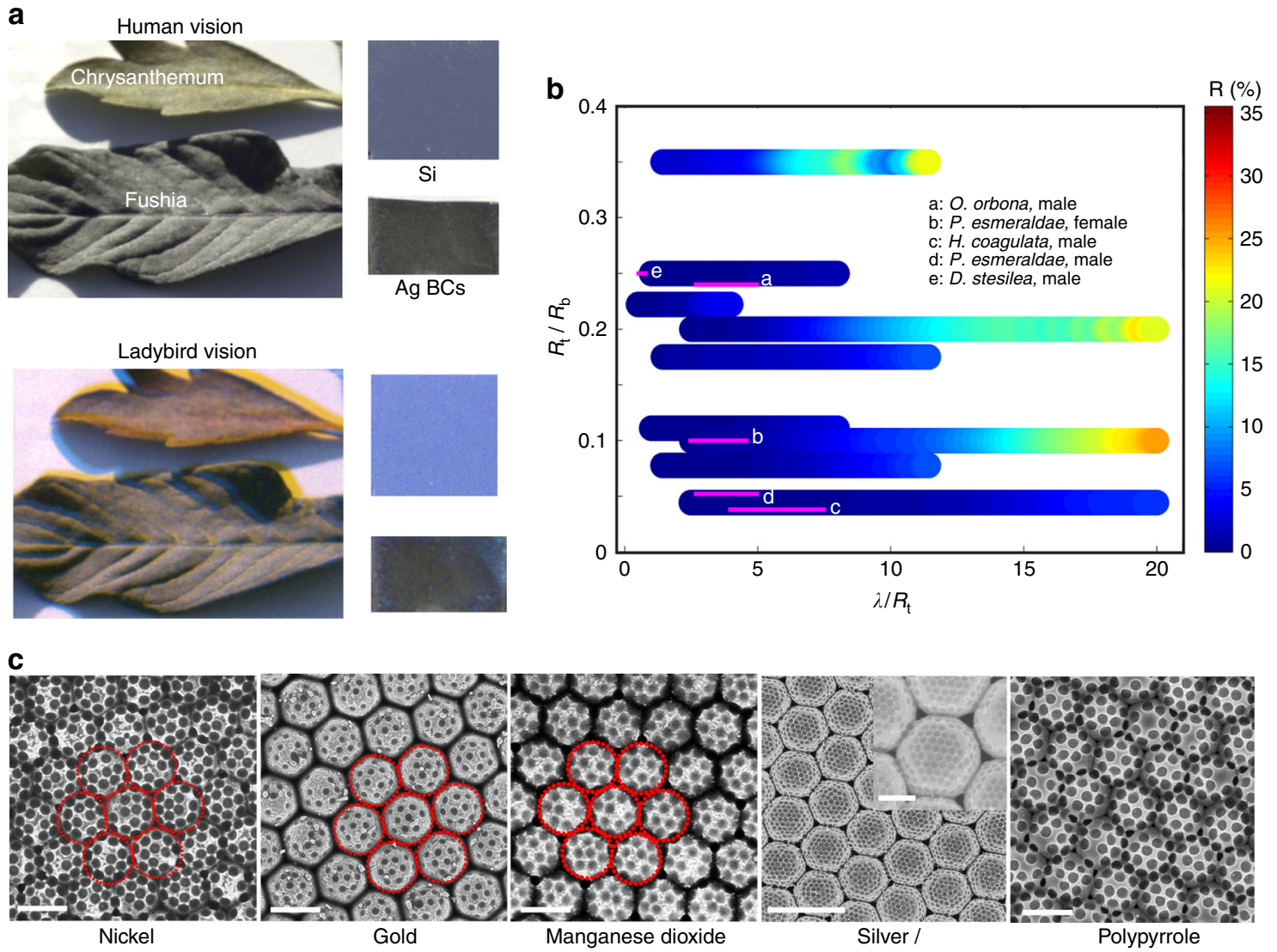

Nickel

Gold

manganese dioxide

Polypyrrole

Fig. 5 Possible camouflage function of BCs and their fabrication in different materials compositions. a Simulated human and ladybird beetle visions of BCs and two different leaf species. $\mathbf{b}$ Comparison of the structural parameters of natural and synthetic brochosomes for their optical reflectance; here, the color bar represents the experimental reflectance measurements of various synthetic BC structures and the magenta lines represent the natural brochosomes (i.e., the visible spectrum of the ladybird over $R_{\mathrm{t}}$ determines the line length and position along the $x$-axis). These natural brochosomes include a Oncometopic orbona, male. b Proconia esmeraldae, female. c Homalodisca coagulata, male. d Proconia esmeraldae, male. e Diestostemma stesilea, male (Supplementary Fig. 24). The observing (incident) angle is $45^{\circ}$. Note that the geometrical parameters of the aforementioned natural brochosomes are located at the low reflection region in the optical reflectance map obtained from the reflectance measurements of the synthetic brochosomes. The normalized plot allows one to identify the corresponding antireflection design parameters of the synthetic brochosome given a specific wavelength of light. c Scanning electron micrographs showing various $\mathrm{BCs}$ fabricated from nickel $(\mathrm{Ni})$, gold $(\mathrm{Au})$, manganese oxide $\left(\mathrm{MnO}_{2}\right)$, silver/manganese oxide $\left(\mathrm{Ag} / \mathrm{MnO}_{2}\right)$, and polypyrrole (PPy). Scale bars: $2 \mu \mathrm{m}\left(\mathrm{Ni}, \mathrm{Au}, \mathrm{MnO}_{2}, \mathrm{PPy}\right)$ and $5 \mu \mathrm{m}\left(\mathrm{Ag} / \mathrm{MnO}_{2}\right.$, inset, $\left.2 \mu \mathrm{m}\right)$

surface. Different amounts of aqueous dispersions composed of PS latex beads ( $2.5 \mathrm{wt} \%$, purchased from Alfa Aesar) were spin-coated onto the treated glass slides (Supplementary Table 1). After dried in the ambient conditions, the glass slide covered by the PS spheres was immersed slowly into a water bath with the addition of $\sim 0.01 \mathrm{~g} \mathrm{~L}^{-1}$ sodium dodecyl sulfate (SDS) at an angle of $\sim 65^{\circ}$ from the normal of the water surface. The PS spheres would assemble into a monolayer film at the air/ water interface. A piece of silicon wafer was used to pick up the free-standing MCC template. Heat treatment at $110^{\circ} \mathrm{C}$ for 3 min on a hot plate was performed to thermally anchor the PS spheres onto the silicon substrate before depositing a thin gold $(\mathrm{Au})$ layer (usually $100 \mathrm{~nm}$ ) onto the MCC template by a sputtering machine. Subsequently, the Au-coated MCC template was plasma-treated for 1 min to create a highly hydrophilic surface. Then, the Au-coated MCC template was used to pick up another layer of free-standing MCC template composed of smaller PS spheres floating at the air/water interface. During the drying process, the small PS spheres would assemble onto the large PS spheres to form a closely packed hexagonal array, leading to the formation of the DCC template.

Preparation of the synthetic brochosomal coatings. The MCC template was prepared by a previously reported spin-coating method ${ }^{25,26}$. Then a thin layer of Au film was evaporated onto the MCC template, which was used as a seed layer to guide the subsequent electrodeposition process. Another layer of MCC template was transferred onto the Au-coated MCC template to form the DCC template. The DCC template was used as the cathode for metal electroplating. The electrolyte for silver plating contained $30 \mathrm{mM}$ silver nitrate and $7 \mathrm{mM}$ SDS. The electrolyte was maintained at a temperature of $45^{\circ} \mathrm{C}$ on a hotplate. The deposition voltage and time was $1.5 \mathrm{~V}$ and $8 \mathrm{~min}$ unless otherwise specified. Electroplating of Au was performed in pure $10 \mathrm{mM}$ gold(III) chloride $\left(\mathrm{HAuCl}_{4}\right)$ solution at $2.5 \mathrm{~V}$ for $5 \mathrm{~min}$. Nickel electrodeposition was carried out in $1.1 \mathrm{M}$ nickel(II) sulfate $\left(\mathrm{NiSO}_{4}\right), 10 \mathrm{mM}$ SDS, and $0.65 \mathrm{M}$ boric acid $\left(\mathrm{H}_{3} \mathrm{BO}_{3}\right)$ with the $\mathrm{pH}$ value adjusted to 3 using sulfuric acid $\left(\mathrm{H}_{2} \mathrm{SO}_{4}\right)$ at $3 \mathrm{~V}$ for $15 \mathrm{~min}$. The DCC template was used as the anode for PPy and metal oxide deposition. For PPy deposition, the electrolyte was formed by $0.1 \mathrm{M}$ pyrrole and $0.1 \mathrm{M}$ sodium dodecylbenzene sulfonate. The deposition voltage for PPy was $1.5 \mathrm{~V}$ for $1 \mathrm{~min}$. Electroplating of $\mathrm{MnO}_{2}$ was conducted in $0.1 \mathrm{M}$ manganese acetate at $3 \mathrm{~V}$ for $4 \mathrm{~min}$. After immersing the electroplated DCC template into dichloromethane for $1 \mathrm{~min}$ to remove the PS spheres, $\mathrm{BCs}$ of the electroplated material are obtained. Step-by-step electroplating can create BCs of hybrid materials. For example, $\mathrm{Ag} / \mathrm{MnO}_{2} \mathrm{BCs}$ were created by electroplating of $\mathrm{Ag}$ for $5 \mathrm{~min}$ and $\mathrm{MnO}_{2}$ for $3 \mathrm{~min}$ using the above electrolytes after dissolving the DCC template using dichloromethane.

Preparation of control samples. The Ag mesoporous films were prepared using MCC template on a piece of Au-coated silicon wafer as the cathode electrode with the same electrolyte for the growth of Ag BCs. The deposition voltage was $1.5 \mathrm{~V}$ and the electroplating time was $3.5 \mathrm{~min}$. The electrodeposited Ag nanoporous film was deposited on a piece of Au-coated silicon wafer in the same electrolyte solution at $1.5 \mathrm{~V}$ for $10 \mathrm{~min}$. The $100-\mathrm{nm}$ thick $\mathrm{Ag}$ mirror was thermally evaporated on a piece of silicon wafer.

Fabrication of black silicon. Black silicon was prepared using a deep reactive ion etching method, according to a previous publication ${ }^{42}$. 
UV-Vis-NIR measurements. The UV-Vis-NIR reflection spectra in the wavelength range of 250-2000 nm were measured (Lambda 950, Perkin-Elmer). The incident angles can be varied from $8^{\circ}$ to $68^{\circ}$.

FDTD simulations. FDTD simulations were carried out using the commercial software Lumerical Solutions. Constrained by the computer resources, we did not consider the roughened sidewall structure of the brochosome, which consisted of numerous Ag nanoparticles $\sim 30$ to $\sim 70 \mathrm{~nm}$ in size. Instead, we built the FDTD model of BCs using smooth Ag shells with ordered pits for simplicity (Supplementary Fig. 15). As a result, our model did not capture the antireflection contributions due to the roughened sidewall of the BCs in the experiments, which is known to effectively suppress reflection ${ }^{29}$. While the use of smooth Ag shells as FDTD models gives rise to significantly increased reflection compared to the experimental measurements, the qualitative trend of the reflection spectra can still be revealed by the FDTD simulation results. The ordered pits were created using the open source software Blender. In this model, we first created an MCC template composed of small spheres in the software. Then, a small force was applied to place the MCC template onto the large PS spheres and simultaneously apply a weak attraction force between the small PS spheres to maintain a close packing of the MCC template. We then increased the diameter of the large PS spheres to the extent that it can bury half of the small PS spheres. Finally, we converted the large PS spheres into Ag shells with a shell thickness equal to the radius of the small PS spheres. We set the materials property of the small PS to be vacuum to better represent the actual $\mathrm{BC}$ configurations in our experiments. The models were then input to the software for FDTD simulations.

UV photography. To mimic the vision of insects and birds, we first modified the camera (Canon T4i) to be UV sensitive by LifePixel (Mukilteo, WA). Then we used a software micaToolbox developed by Troscianko and Stevens to simulate the vision as observed by insects and birds ${ }^{35}$. To create the simulated images, we first used the UV and regular camera to take photographs on the Ag BCs lying on a green leaf under sunlight, respectively. Then the toolbox converted the photos to multispectral objective images consisting of all RGB and UV channels. Finally, the false-color-simulated images were produced based on the specific spectral sensitivities of the visual system of a given insect, as shown in Fig. 5 and Supplementary Fig. 23.

Evaluation of color difference in non-human visual systems. We used a published method for comparing colors in non-human visual systems, which is based on Vorobyev and Osorio's receptor noise model ${ }^{34,43}$ implemented in MICA. This method calculates the distance between two colors with the unit JND (just noticeable difference). This unit is based on the signal-to-noise ratios of each channel of the specific animal visual system. If the JND value between two colors is less than 1 , then the images are said to be indistinguishable. However, if the value is above $\sim 3$, then the images are said to be discriminable under good lighting conditions $^{44}$. The Weber fraction (i.e., the ratio of intensity between two lights perceived by a visual system at threshold ${ }^{44}$ used is 0.05 based on ladybird beetle and human's visual system ${ }^{36,45}$. Data are measured from 32-bit normalized objective images processed by MICA Toolbox.

Data availability. The data that support the findings of this study are available from the corresponding authors upon reasonable request.

Received: 5 March 2017 Accepted: 14 September 2017

Published online: 03 November 2017

\section{References}

1. Miller, W. H., Bernard, G. D. \& Allen, J. L. The optics of insect compound eyes. Science 162, 760-767 (1968).

2. Clapham, P. B. \& Hutley, M. C. Reduction of lens reflection by moth eye principle. Nature 244, 281-282 (1973).

3. Srinivasarao, M. Nano-optics in the biological world: beetles, butterflies, birds, and moths. Chem. Rev. 99, 1935-1961 (1999).

4. Barthlott, W. \& Neinhuis, C. Purity of the sacred lotus, or escape from contamination in biological surfaces. Planta 202, 1-8 (1997).

5. Autumn, K. et al. Adhesive force of a single gecko foot-hair. Nature 405, 681-685 (2000).

6. Gao, X. F. \& Jiang, L. Water-repellent legs of water striders. Nature 432, 36-36 (2004).

7. Parker, A. R. \& Townley, H. E. Biomimetics of photonic nanostructures. Nat. Nanotechnol. 2, 347-353 (2007).

8. Vukusic, P., Hallam, B. \& Noyes, J. Brilliant whiteness in ultrathin beetle scales. Science 315, 348-348 (2007).

9. Wong, T. S. et al. Bioinspired self-repairing slippery surfaces with pressurestable omniphobicity. Nature 477, 443-447 (2011).
10. Liu, K. S., Yao, X. \& Jiang, L. Recent developments in bio-inspired special wettability. Chem. Soc. Rev. 39, 3240-3255 (2010).

11. Boesel, L. F., Greiner, C., Arzt, E. \& del Campo, A. Gecko-Inspired surfaces: a path to strong and reversible dry adhesives. Adv. Mater. 22, 2125-2137 (2010).

12. Jeong, K. H., Kim, J. \& Lee, L. P. Biologically inspired artificial compound eyes. Science 312, 557-561 (2006).

13. Kolle, M. et al. Mimicking the colourful wing scale structure of the Papilio blumei butterfly. Nat. Nanotechnol. 5, 511-515 (2010).

14. Tulloch, G. S., Shapiro, J. E. \& Cochran, G. W. The occurrence of ultramicroscopic bodies with leafhoppers and mosquitoes. Bull. Brooklyn Entomol. Soc. 47, 41-42 (1952).

15. Tulloch, G. S. \& Shapiro, J. E. Brochosomes. Bull. Brooklyn Entomol. Soc. 48, 57-63 (1953)

16. Tulloch, G. S. \& Shapiro, J. E. Brochosomes and leafhoppers. Science 120, 232-232 (1954).

17. Day, M. F. \& Briggs, M. The origin and structure of brochosomes. J. Ultrastruct. Res. 2, 239-244 (1958).

18. Rakitov, R. A. Powdering of egg nests with brochosomes and related sexual dimorphism in leafhoppers (Hemiptera: Cicadellidae). Zool. J. Linn. Soc. 140, 353-381 (2004).

19. Rakitov, R. \& Gorb, S. N. Brochosomes protect leafhoppers (Insecta, Hemiptera, Cicadellidae) from sticky exudates. J. R. Soc. Interface 10, 20130445 (2013).

20. Rakitov, R. \& Gorb, S. N. Brochosomal coats turn leafhopper (Insecta, Hemiptera, Cicadellidae) integument to superhydrophobic state. Proc. R. Soc. B Biol. Sci. 280, 20122391 (2013)

21. Swain, R. B. Notes on the oviposition and life history of the leafhopper Oncometopta undata Fabr. (Homoptera: Cicadellidae). Entomol. News 47, 264-266 (1936).

22. Chattopadhyay, S. et al. Anti-reflecting and photonic nanostructures. Mater. Sci. Eng. R 69, 1-35 (2010).

23. Hix, R. L. Egg-laying and brochosome production oberved in glassy-winged sharpshooter. Calif. Agricult. 55, 19-22 (2001).

24. Rakitov, R. A. in Functional Surfaces in Biology Vol 1, (ed. Gorb, S. N.) 113-137 (Springer, Dordrecht, 2009).

25. Yang, S. K., Cai, W. P., Kong, L. C. \& Lei, Y. Surface nanometer-scale patterning in realizing large-scale ordered arrays of metallic nanoshells with well-defined structures and controllable properties. Adv. Funct. Mater. 20, 2527-2533 (2010).

26. Lei, Y., Yang, S. K., Wu, M. H. \& Wilde, G. Surface patterning using templates: concept, properties and device applications. Chem. Soc. Rev. 40, 1247-1258 (2011).

27. Gao, P. Q. et al. Large-area nanosphere self-assembly by a micro-propulsive injection method for high throughput periodic surface nanotexturing. Nano Lett. 15, 4591-4598 (2015).

28. Velikov, K. P., Christova, C. G., Dullens, R. P. A. \& van Blaaderen, A. Layer-bylayer growth of binary colloidal crystals. Science 296, 106-109 (2002).

29. Teperik, T. V. et al. Omnidirectional absorption in nanostructured metal surfaces. Nat. Photonics 2, 299-301 (2008).

30. Ibn-Elhaj, M. \& Schadt, M. Optical polymer thin films with isotropic and anisotropic nano-corrugated surface topologies. Nature 410, 796-799 (2001).

31. Aydin, K., Ferry, V. E., Briggs, R. M. \& Atwater, H. A. Broadband polarizationindependent resonant light absorption using ultrathin plasmonic super absorbers. Nat. Commun. 2, 517 (2011).

32. Campbell, P. Enhancement of light-absorption from randomizing and geometric textures. J. Opt. Soc. Am. B 10, 2410-2415 (1993).

33. Branz, H. M. et al. Nanostructured black silicon and the optical reflectance of graded-density surfaces. Appl. Phys. Lett. 94, 231121 (2009).

34. Osorio, D. \& Vorobyev, M. Photoreceptor spectral sensitivities in terrestrial animals: adaptations for luminance and colour vision. Proc. R. Soc. B Biol. Sci. 272, 1745-1752 (2005)

35. Troscianko, J. \& Stevens, M. Image calibration and analysis toolbox-a free software suite for objectively measuring reflectance, colour and pattern. Methods Ecol. Evol. 6, 1320-1331 (2015).

36. Lin, J. T. Identification of photoreceptor locations in the compound eye of Coccinella septempunctata Linnaeus (Coleoptera, Coccinellidae). J. Insect Physiol. 39, 555-562 (1993).

37. Wei, W. F., Cui, X. W., Chen, W. X. \& Ivey, D. G. Manganese oxide-based materials as electrochemical supercapacitor electrodes. Chem. Soc. Rev. 40, 1697-1721 (2011).

38. Li, C., Bai, H. \& Shi, G. Q. Conducting polymer nanomaterials: electrosynthesis and applications. Chem. Soc. Rev. 38, 2397-2409 (2009).

39. Zhou, L. et al. Self-assembly of highly efficient, broadband plasmonic absorbers for solar steam generation. Sci. Adv. 2, e1501227 (2016).

40. Lin, Q. F. et al. Inverted nanocone-based thin film photovoltaics with omnidirectionally enhanced performance. ACS Nano 8, 6484-6490 (2014). 
41. Yang, S. K., Dai, X. M., Stogin, B. B. \& Wong, T. S. Ultrasensitive surfaceenhanced Raman scattering detection in common fluids. Proc. Natl Acad. Sci. USA 113, 268-273 (2016).

42. Kang, C. K. et al. The fabrication of patternable silicon nanotips using deep reactive ion etching. J. Micromech. Microeng. 18, 075007 (2008).

43. Vorobyev, M. \& Osorio, D. Receptor noise as a determinant of colour thresholds. Proc. R. Soc. B Biol. Sci. 265, 351-358 (1998).

44. Siddiqi, A., Cronin, T. W., Loew, E. R., Vorobyev, M. \& Summers, K. Interspecific and intraspecific views of color signals in the strawberry poison frog Dendrobates pumilio. J. Exp. Biol. 207, 2471-2485 (2004).

45. Hofer, H., Carroll, J., Neitz, J., Neitz, M. \& Williams, D. R. Organization of the human trichromatic cone mosaic. J. Neurosci. 25, 9669-9679 (2005).

\section{Acknowledgements}

We thank Josh Stapleton from Materials Characterization Laboratory and Leland Shawn Burghard at The Pennsylvania State University for the help with the optical measurements and the greenhouse management, respectively. We acknowledge funding support by the Defense Advanced Research Projects Agency Award\# D14AP00042 (materials and optical characterizations), the National Science Foundation CAREER Award\# 1351462 (materials fabrication), Start-Up Fund from The Pennsylvania State University, and Wormley Family Early Career Professorship. Stogin acknowledges support from the NSF Graduate Research Fellowship (Grant No.: DGE1255832). Publication of this article was funded in part by The Pennsylvania State University Libraries Open Access Publishing Fund. Part of the work was conducted at the Penn State node of the NSF-funded National Nanotechnology of Infrastructure Network.

\section{Author contributions}

S.Y. and T.-S.W. designed the experiments. S.Y. carried out the materials fabrication, wetting, and optical characterizations. S.Y., N.S., B.B.S., J.W., and Y.H. conducted the data analysis. N.S. and J.W. performed vision simulations of various organisms and experimental verifications. S.Y., B.B.S., and T.-S.W. wrote the paper. All authors contributed to paper revision.

\section{Additional information}

Supplementary Information accompanies this paper at doi:10.1038/s41467-017-01404-8.

Competing interests: The authors declare no competing financial interests.

Reprints and permission information is available online at http://npg.nature.com/ reprintsandpermissions/

Publisher's note: Springer Nature remains neutral with regard to jurisdictional claims in published maps and institutional affiliations.

(c) (i) Open Access This article is licensed under a Creative Commons Attribution 4.0 International License, which permits use, sharing, adaptation, distribution and reproduction in any medium or format, as long as you give appropriate credit to the original author(s) and the source, provide a link to the Creative Commons license, and indicate if changes were made. The images or other third party material in this article are included in the article's Creative Commons license, unless indicated otherwise in a credit line to the material. If material is not included in the article's Creative Commons license and your intended use is not permitted by statutory regulation or exceeds the permitted use, you will need to obtain permission directly from the copyright holder. To view a copy of this license, visit http://creativecommons.org/ licenses/by/4.0/.

(C) The Author(s) 2017 